\title{
Efficacy of autologous leukocyte-reduced platelet-rich plasma therapy for patellar tendinopathy in a rat treadmill model
}

\author{
Mamoru Yoshida \\ Hiroki Funasaki \\ Keishi Marumo
}

Department of Orthopaedic Surgery, The Jikei University School of Medicine, Tokyo, Japan

Corresponding author:

Mamoru Yoshida

Department of Orthopaedic Surgery,

The Jikei University School of Medicine

3-25-8, Nishi-Shinbashi, Minato-ku

105-8461 Tokyo, Japan

E-mail: mamoru@jikei.ac.jp

\section{Summary}

Background: An autologous platelet-rich plasma (PRP) therapy has currently been applied for the tendinopathy; however, its efficacy and an optimal platelets concentration in PRP were uncertain. We analyzed them in an animal model prepared using a repetitive running exercise.

Methods: We made the tendinopathy rat model of patellar tendon using a rodent treadmill machine. Rats with tendinopathy were injected with leukocyte-reduced PRP at the platelets concentration of $1.0 \times 10^{6} / \mu \mathrm{L}$ (P10 group), PRP at the platelets concentration of $5.0 \times 10^{5} / \mu \mathrm{L}$ (P5 group) or normal saline (control group) into the space between the patellar tendon and the fat pad bilaterally or were multiply dry-needled at the tibial insertion site (MN group) at once. To assess the pain-reliving effect, the spontaneous locomotor activities at night (12 h) were measured every day. Histological sections of the patellar tendon stained with hematoxylineosin or prepared by TdT-mediated dUTP nick end labeling were microscopically analyzed.

Results: The numbers of spontaneous locomotor activities in the P10 group were significantly larger than those in the P5, MN or control groups and they recovered up to a healthy level. On histologic examinations, the numbers of microtears, laminations, or apoptotic cells in the patellar tendons in the P10 or P5 groups were significantly lower than those in the MN or control groups, although no significant differences were observed between the P10 and P5 groups.
Conclusions: The injections of an autologous leukocyte-reduced PRP were effective for pain relief and for partial restoration of the patellar tendon in the tendinopathy rat model. The injections of a PRP at the platelets concentration of $1.0 \times 10 \% / \mu \mathrm{L}$ completely relieved the pain and were more effective than those at the platelets concentration of $5.0 \times 105 / \mu \mathrm{L}$ whereas there was no difference for the effect of histological restoration or apoptosis inhibition between them.

KEY WORDS: platelet-rich plasma, rat, tendinopathy.

\section{Introduction}

Tendinopathy is the most common tendon disorder characterized by activity-related pain, focal tendon tenderness and decreased strength and movement in the affected area. Tendinopathy can occur in almost any tendon and if developed, it impairs active performance in athletes and workers of many occupations that involve repetitive movements. Some of the most common tendinopathies are tennis elbow, jumper's knee and Achilles tendinopathy ${ }^{1}$.

In the current decade, a therapy of autologous platelet-rich plasma (PRP) has been clinically applied for the treatment of the tendinopathy as tennis elbow, jumper's knee, Achilles tendinopathy, plantar fasciitis and other tendinopathies. Significant therapeutic effects of PRP were showed for lateral or medial epicondylitis in elbows, jumper's knee and Achilles tendinopathy compared to the conventional therapies as the injection of corticosteroid or a focused shockwave therapy ${ }^{2-12}$. On the other hand, there were some clinical examinations which showed no significant effects of PRP for the treatment of Achilles tendinopathy ${ }^{13}, 14$. However, there were few high-level literature as randomized controlled trials regarding the application of PRP in the management of patellar and Achilles tendinopathy. An efficacy and effectiveness of PRP therapy were still uncertain for the treatment of patellar or Achilles tendinopathy at the present.

The majority of the basic research has demonstrated a beneficial effect of PRP on the proliferation of tenocytes, collagen synthesis and the tendon healing process $^{15}$. In the tendinopathy animal model, Dallaudière demonstrated that a mono-injection of PRP improved tendon healing with no local toxicity in a rat collagenase-induced tendinopathy of patellar or 
Achilles tendon ${ }^{16}$ and Chen demonstrated that a PRP treatment resulted in better healing of injured tendons with improved histological outcomes and biomechanical functions in a rat collagenase-induced tendinopathy of Achilles tendon ${ }^{17}$. As the animal model for the patellar tendinopathy, it was suggested that a model prepared using a repetitive running exercise on a rodent treadmill machine pathophysiologically reproduced well an actual tendinopathy compared to the collagenase-induced model, since there was no inflammation reaction in/around the tendon in actual tendinopathy although the inflammation was observed in/around the tendon in the collagenase-induced tendinopathy and the clinical tendinopathy was pathogenetically induced with the over-use and/or over-load stress like a repetitive running exercise for a long distance ${ }^{18-21}$. The previous basic researches described above and also the current animal study 22 were performed using the collagenase-induced model and there was no examination, which examined the therapeutic effect of PRP for the tendinopathy in an animal model prepared using a repetitive running exercise on a rodent treadmill machine to our knowledge at the present. We have experienced to make this animal model and have examined the study using the rat tendinopathy model prepared by a repetitive running exercise 23 .

Regarding the optimal concentration of the platelets in PRP for the tendon healing, some researchers reported in vitro studies. McCarrell demonstrated that the PRP at the platelets concentration of $1.2 \times 10^{6} / \mu \mathrm{L}$ led to a paradoxical effect on collagen synthesis on the culture of horse tendons compared to the PRP at the intermediate concentration of the platelets, while also presenting an increase in inflammatory mediators $^{24}$. Boswell also demonstrated that the PRP at the high platelets concentration reduced tendon metabolism and collagen synthesis in equine tendon culture $^{25}$. Moreover, Giusti demonstrated that the PRP at the platelets concentration between $5.0 \times 10^{5} / \mu \mathrm{L}$ and $1.0 \times 10^{6} / \mu \mathrm{L}$ stimulated cell proliferation and migration as well as collagen production in human tenocyte cultures while higher platelet concentrations have an inhibitive effect both in terms of cell metabolism and collagen synthesis ${ }^{26}$. From those information it seemed that the platelets concentration between $5.0 \times 10^{5} / \mu \mathrm{L}$ and $1.0 \times 10^{6} / \mu \mathrm{L}$ in PRP was optimal for the tendon healing. There was no examination which analyzed the difference for the efficacy of a PRP therapy at the different platelets concentration in the tendinopathy animal model. The optimal concentration of the platelets in PRP was still uncertain for the therapy of the tendinopathy.

Regarding the influence of the leukocytes in PRP against the metabolism of tenocytes, Dragoo demonstrated that PRPs rich in leukocytes led to a significant inflammatory cellular reaction in rabbit tendons at 5 days after PRP infiltration ${ }^{27}$. McCarrell demonstrated that the PRP with a high concentration of leukocytes led to higher expression of pro-inflammatory cytokines and catabolic mediators as well as low- er collagen synthesis ${ }^{24}$. It appeared that the leukocyte inhibited the restoration process for the treatment of the tendinopathy since the inflammation damaged the extracellular matrix and a lower synthesis of the collagen molecules suppressed the conformation of the tendon matrix. Hence, we used a leukocyte-reduced PRP for the present study.

We investigated the therapeutic effect of an autologous leukocyte-reduced PRP for tendinopathy in a rat model prepared using a repetitive running exercise on a rodent treadmill machine in the present study. An efficacy for a pain-relieving or a histological tendon tissue-repair of PRP was scientifically analyzed. Moreover, we evaluated the therapeutic effects of an autologous PRP at the different concentration of the platelets between $5.0 \times 10^{5} / \mu \mathrm{L}$ and $1.0 \times 10^{6} / \mu \mathrm{L}$ in the same manner. Furthermore, we analyzed the effectiveness of a multiple dry-needling performed at the tendinopathy site in order to know its contribution for the treatment of the tendinopathy. The present study was designed and underwent according to the journal's editorial 28 .

\section{Materials and methods}

\section{Reagents and materials}

The autologous PRP preparation system, MyCells, was purchased from Kaylight, Tel Aviv, Israel. The normal saline for injections was from Otsuka Pharmaceutical Factory, Inc., Naruto, Japan. A rodent treadmill, KN-73, was purchased from Natsume Seisakusho, Co., Ltd., Tokyo, Japan. The Supermex system for measurement of spontaneous locomotor activities of rats was from Muromachi Kikai, Tokyo, Japan. The ApopTag Peroxidase In Situ Apoptosis Detection Kit was from EMD Millipore Corporation, Billerica, MA, USA.

\section{Animals}

Male Wistar rats (16-18 weeks of age, weighing 350$400 \mathrm{~g}$ ) were purchased from Nippon SLC, Hamamatsu, Shizuoka, Japan, and served as subjects in the study. Rats were housed in an environmentally controlled animal facility on a 12:12 light/dark cycle with food and water available ad libitum in the laboratory animal section.

\section{The animal model}

All experiments were conducted in accordance with the institutional guidelines for the care and use of experimental animals after an institutional approval for the study was obtained. A rat model of patellar tendinopathy was made according to the method of Flatow et al. ${ }^{18-21,23}$. A running exercise was performed on a rodent treadmill with $10 \%$ inclination of the running protocol. The rats were acclimated to the treadmill by gradually increasing running speed and 
time for 2 weeks before the running exercise. Running distance reached up to $40 \mathrm{~km}$ during 40 days and the mean running speed was $0.5 \mathrm{~km} / \mathrm{h}$. The spontaneous locomotor activities at night were measured for $12 \mathrm{~h}$ with the Supermex system on the day before the running exercise and on the day after exercise. The reduction in spontaneous locomotor activity after exercise was different among rats ${ }^{23}$. The rats showing $>25 \%$ reduction $(25-33 \%)$ in spontaneous locomotor activity after exercise were then selected as the tendinopathy rats for later study. Approximately $85 \%$ of the rats ran the whole distance and approximately $90 \%$ of them revealed $>25 \%$ reduction in locomotor activities. In pre-study, we made histological sections of the patellar tendons or rotator cuffs in the shoulders in ten rats, which revealed $>25 \%$ reduction in locomotor activities or in five rats which revealed no reduction in activities after running exercise. Then, the tendinopathy-specific histopathological changes ${ }^{29}$ were observed at the insertion site of the patellar tendon with light microscopy in all ten activity-reduced rats (Fig. 3) whereas no tendinopathy-specific histopathological changes were observed in five activitynon-reduced rats. Moreover, less tendinopathy-specific histopathological changes were observed at the insertion site of the rotator cuffs in shoulders in both rats. We finally made 30 tendinopathy rats for the present study from 50 rats.

\section{Group division}

The rats with tendinopathy were divided into four groups. The rats in the P10 group, the P5 group or the control group were injected with autologous leukocyte-reduced PRP at the platelets concentration of $1.0 \times 10^{6} / \mu \mathrm{L}$, autologous leukocyte-reduced PRP at the platelets concentration of $5.0 \times 10^{5} / \mu \mathrm{L}$ or normal saline, respectively, into the spaces between the patellar tendons and fat pad tissues bilaterally. Each group consisted of eight rats. Rats in fourth, the multiple dry-needling (MN) group, were pin-pricked ten times using a $27 \mathrm{G}$ injection needle at the tibial insertional site of the patellar tendon without injections. The MN group consisted of six rats.

\section{Observation of general status and body weight measurements}

The general status was observed once a day from the day before the running exercise started to the end of the study. Body weight was measured at every 5 days during the study period.

\section{Preparation of autologous platelet-rich plasma}

We used an autologous PRP preparation system, MyCells, to obtain a rat autologous leukocyte-reduced PRP since the system could prepare the leukocytereduced $\mathrm{PRP}$ at high platelets concentration and it was hard to purchase the other PRP preparation kits like Biomet GPSIII, Arteriocyte Magellan or PRGF in our nation. A whole blood of $10 \mathrm{~mL}$ was aspirated from the hearts in 5 rats and a leukocyte-reduced PRP of $2.0 \mathrm{~mL}$ was obtained by using a PRP preparation system according to the manufacture's instruction. Briefly, a whole blood of $10 \mathrm{~mL}$ was inserted into the separation spit of the system and it was centrifuged for 7 minutes at $2000 \times \mathrm{g}$. After aspiration of the supernatant plasma, the residual plasma of 2.0 $\mathrm{mL}$ was pipetting well in order to dissolve the platelets which were precipitated on the separation gel. Then, the filter column was inserted into the separation spit to exclude the debris and a filtered nonactivated PRP of $2.0 \mathrm{~mL}$ was obtained by aspiration from the filtration column. A hundred $\mu \mathrm{L}$ of a prepared PRP was mixed in the normal saline at the final volume of $1.0 \mathrm{~mL}$ and the number of the platelets or leukocytes was counted. We obtained a rat autologous PRP at the platelets concentration of $1.3 \times 10^{6} / \mu \mathrm{L}$ containing the leukocytes at the approximate half concentration of the one in whole blood and no erythrocytes. An obtained PRP was classified as type 3 in Mishra's classification and was classified as pure PRP in PAW classification since we did not activate a PRP before injection. Then, we made a PRP of $1.0 \mathrm{~mL}$ at the platelets concentration of $1.0 \times 10^{6} / \mu \mathrm{L}$ or $5.0 \times 10^{5} / \mu \mathrm{L}$, respectively, for further examinations.

\section{Injections of autologous PRP or normal saline in- to hind paws}

After general anesthesia with $2.5 \%$ isoflurane, the fur at the knee joints was shaved and $25 \mu \mathrm{L}$ of autologous leukocyte-reduced PRP or normal saline was injected into the space between the patellar tendon and the fat pad tissue at the distal area of the patellar tendon under ultrasonography (US) guidance using a 0.5 $\mathrm{mL}$ injection syringe and a $27 \mathrm{G}$ injection needle from lateral side of the knees, respectively, in the P10, P5 or control groups. Before injection the platelets in PRP were not activated. Since there was a possibility that the direct injection into the tendon produced the tears or laminations in the tendon, we avoided the direct injection into the tendon and selected a space between tendon and fat pad as a target for injection. In pre-study, we injected $30 \mu \mathrm{L}$ of PRP or saline containing the indigo carmine dye into the space between patellar tendon and fat pad in ten rats and we confirmed that there was no damage in the tendon and no leakage into the cavity of knees in ten rats by the direct inspection after surgical open of the knees $^{23}$. Therefore, we injected $25 \mu \mathrm{L}$ of PRP or saline for more prevention of the leakage. Then, one technician practiced the injection of PRP or normal saline containing the indigo carmine dye for twenty times under ultrasonography (US) guidance followed by confirmation whether there was a leakage or not with the direct inspection after surgical open of the knees. After the technician mastered the way of the injection, the injections were performed once at the next day following completion of the running exercise (Fig. 1). 


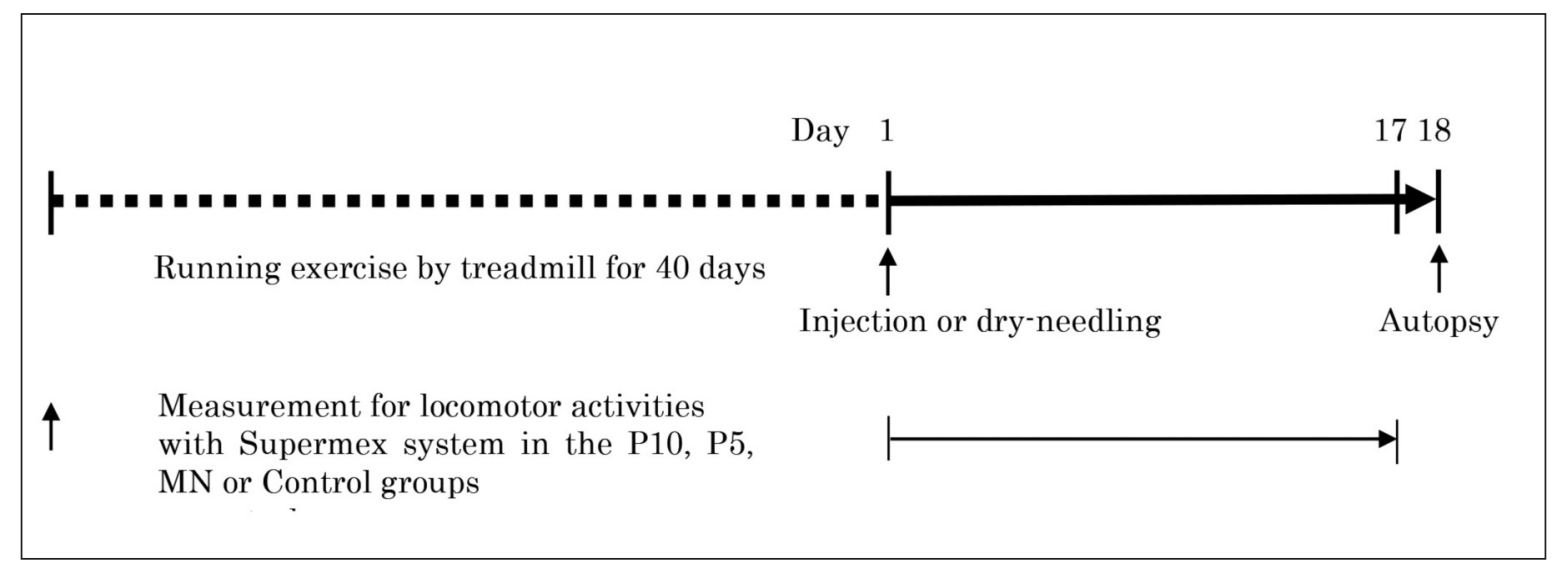

Figure 1. The study protocol.

\section{Measurements of spontaneous locomotor activities}

Spontaneous motor activity of each rat was examined using the Supermex system for $12 \mathrm{~h}$ each night for 17 days after injections of PRP or saline or multiple dryneedling in all groups. The Supermex system could quantify the locomotor activities, which reflected the degree of the pain in limbs of rats. Thus, the degree of pain-relief in the knee could be quantified by the measurement of the locomotor activities. The system was used in many animal studies, in which the degree of the pain-relief was quantitatively measured, and the reliability of the system was highly evaluated comparing to other methods in the literatures ${ }^{23}, 30-33$. Details of spontaneous locomotor activity measurement have been described previously $23,30-33$. Briefly, spontaneous locomotor activities were determined by detecting the movement of infrared radiation emitted from each animal. The activities were measured as a single count when an animal moved from one region of the measurement area divided optically by multiple lenses, to a neighboring region. Total counts were calculated by summing up all counts of 10-min periods. The infrared filter with $25 \%$ signal reduction was used to remove the noise of the grooming motion. The relative number of motor activities in each group was calculated and the mean of the relative changes was plotted on a graph in order to evaluate the relative changes for motor activities of rats during the experiment period, since the absolute values for motor activities were different among rats, which were $9787 \pm 1982$ (6703-14191) counts for 50 healthy rats. A statistical analysis was performed to examine the outcome differences between each group.

\section{Histological examinations}

The knee tissue samples were obtained from 6 healthy normal rats and 6 tendinopathy rats at day 1 (controls), and from 6 rats in the P10, the P5, the MN or the control groups, respectively, at day 18. Following inhalation with $5 \%$ isoflurane, the rats were killed by immediate blood draw. Whole knee joints were fixed in $4 \%$ paraformaldehyde at $\mathrm{pH} 7.4$ for 3 days, and decalcified in $20 \%$ EDTA solution for 21 days at $4^{\circ} \mathrm{C}$, and then embedded in paraffin wax. Whole knee joints were sectioned in the sagittal plane with a section width of $5 \mu \mathrm{m}$. The sections were stained with hematoxylin-eosin (H\&E) for histology. They were assessed with a histopathological score determined by counting the number of microtears and laminations located at the insertion site of the patellar tendon as a point in high-power (magnification $200 x$ ) fields in each five sections of tendinopathy rats or $\mathrm{P} 10, \mathrm{P} 5, \mathrm{MN}$ or control groups. A point in the score was doubled in cases where the length of tears or laminations were longer than half the diameter of the visual high-power field (magnification $200 x$ ) on the microscope ${ }^{23}$. The histological sections were also prepared by TdT-mediated dUTP nick end labeling using the ApopTag Peroxidase In Situ Apoptosis Detection Kit according to the manufacturer's instructions. Tendon cells for apoptosis were quantified by counting the number of TUNEL-positive cells in high-power (magnification 400x) fields at the joint side of the tibial insertion site for the patellar tendon in each five sections of tendinopathy rats or P10, P5, $\mathrm{MN}$ or control groups. Three researchers counted the histopathological score in H\&E sections and the number of apoptosis cells in all samples respectively. The standard deviations of these scores or numbers in each histological sample among three researchers were within $5 \%$. The data values were expressed as the mean of the differences among groups after statistical analysis ${ }^{23}$.

\section{Statistical analysis}

Comparative series were considered independent of each other. A mean value and standard deviation was calculated for each daily locomotor activity in each group or for the histopathological score and the cell number of apoptosis in each group. We compared the daily locomotor activities, the histopathological scores and the numbers of apoptosis among groups. Statistical analysis was done using the Wil- 
Table I. Body weight gain.

\begin{tabular}{|c|c|c|c|c|c|}
\hline \multirow[t]{2}{*}{ Group } & \multirow[t]{2}{*}{$\mathbf{n}$} & & \multicolumn{3}{|c|}{ Days after running exercise } \\
\hline & & 0 & 5 & 10 & 15 \\
\hline Control & 8 & $421 \pm 19$ & $426 \pm 21$ & $434 \pm 23$ & $442 \pm 26$ \\
\hline P10 & 8 & $422 \pm 21$ & $425 \pm 22$ & $433 \pm 23$ & $441 \pm 24$ \\
\hline P5 & 8 & $421 \pm 18$ & $425 \pm 20$ & $432 \pm 21$ & $439 \pm 23$ \\
\hline MN & 6 & $418 \pm 20$ & $424 \pm 21$ & $432 \pm 23$ & $440 \pm 25$ \\
\hline
\end{tabular}

Body weight $(\mathrm{g})$ was measured at indicated days. The data indicate the mean \pm standard deviation of ten rats.

The P10, P5 or MN groups was compared with the control group using unpaired $t$ test.

coxon's matched-pairs signed-rank test. A probability value of $<0.05$ was considered statistically significant.

\section{Results}

\section{General status and body weight}

There were no abnormalities observed in any of the groups during the experiment period. The change of body weight during the injection period is shown in Table I. All groups showed a similar trend of body weight changes compared to the control group.

\section{Spontaneous locomotor activities}

The counts for the spontaneous locomotor activities in the P10 group increased up to approximate 1.4fold at day 3 and were maintained, not decreased, for 15 days until the end of the study without any additional PRP injections. Spontaneous motor activities in the $\mathrm{P} 10$ group recovered up to the original level as before commencement of the running exercise. On the other hand, the counts for the spontaneous locomotor activities in the P5 group increased up to approximate 1.2-fold at day 3 and were maintained, not decreased, for 15 days until the end of the study without any additional PRP injections. There were significantly more counts for the spontaneous locomotor activities in the $\mathrm{P} 10$ group than in the $\mathrm{P} 5$, the $\mathrm{MN}$ or the control groups $(p<0.05)$ and there were significantly more counts for the spontaneous locomotor activities in the P5 group than in the MN or the control groups $(p<0.05)$ during 16 days of the experimental periods from day 2 until the end of the study. The counts of the locomotor activities in the $\mathrm{MN}$ and the control groups did not significantly increase and remained in the range of 80 to $120 \%$ of the initial activities. No significant differences were observed for the counts of locomotor activities between the MN group and the control group. The counts for the spontaneous locomotor activities in all groups displayed a trend to gradually increase up along with the days during experimental periods, although no statistical significances were observed (Fig. 2).

\section{Histological findings}

Two major pathological findings were found at the tibial insertion sites of the patellar tendons in tendinopathy rats in all the histological sections stained with hematoxylin-eosin. They were microtears in the tendon body and lamination, i.e., several longitudinal disruptions between the fiber bundle layers (Fig. 3). The same pathological findings were found at the tibial insertion sites of the patellar tendon at day 18 in the P10, P5, MN or control groups in the histological knee sections stained with hematoxylin-eosin (Fig. 4). However, the histopathological scores determined by the number and length of the microtears and laminations in five rats of the $\mathrm{P} 10$ or P5 groups were significantly lower than those in five rats of the MN or control groups $(p<0.05)$ (Tab. II, Fig. 4), indicating that the reactions of tendon repair in the P10 or P5 groups were progressed compared to those in the MN or control groups. No significant differences were observed in the histopathological scores between the P10 group and the P5 group or among tendinopathy rats, MN group and control group (Tab. II). TUNELpositive tendon cells were observed at the joint side of the tibial insertion site for the patellar tendon in all tendinopathy rats. The number of TUNEL-positive cells at the same area in five rats of the P10 or P5 groups was significantly lower than those in five rats of the tendinopathy rats, MN group or control group at day 18 ( $p<0.05$ ) (Tab. III, Fig. 5). No significant differences were observed in the number of TUNELpositive cells between the P10 group and the P5 group or among tendinopathy rats, MN group and control group (Tab. III, Fig. 5).

\section{Discussion}

In the present study, we created an insertional tendinopathy model of the patellar tendon in rats and examined the therapeutic effects of autologous leukocyte-reduced PRP injections. The following findings were obtained: (1) the injections of autologous leukocyte-reduced PRP into the space between the patellar tendon and the fat pad tissue significantly increased the number of spontaneous locomotor activi- 


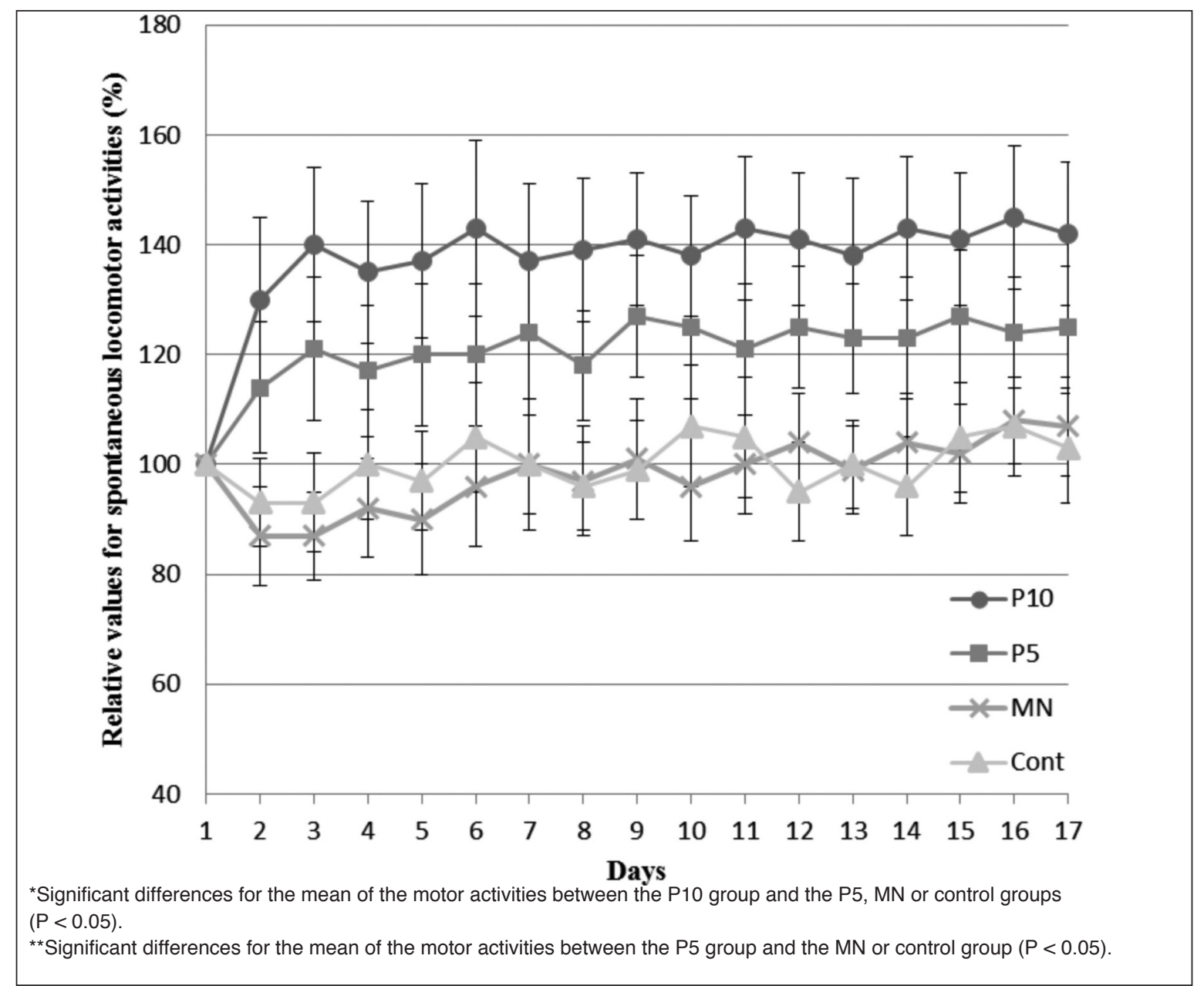

Figure 2. Relative values for spontaneous locomotor activities in the P10, P5, MN or control groups. The spontaneous locomotor activities in rats were examined using a Supermex system at each night for $12 \mathrm{~h}$, every day for 17 days, after completion of the running exercise and after commencement of the injection protocol. The relative number of locomotor activities in each group was calculated and the means or the SD were plotted on a graph.
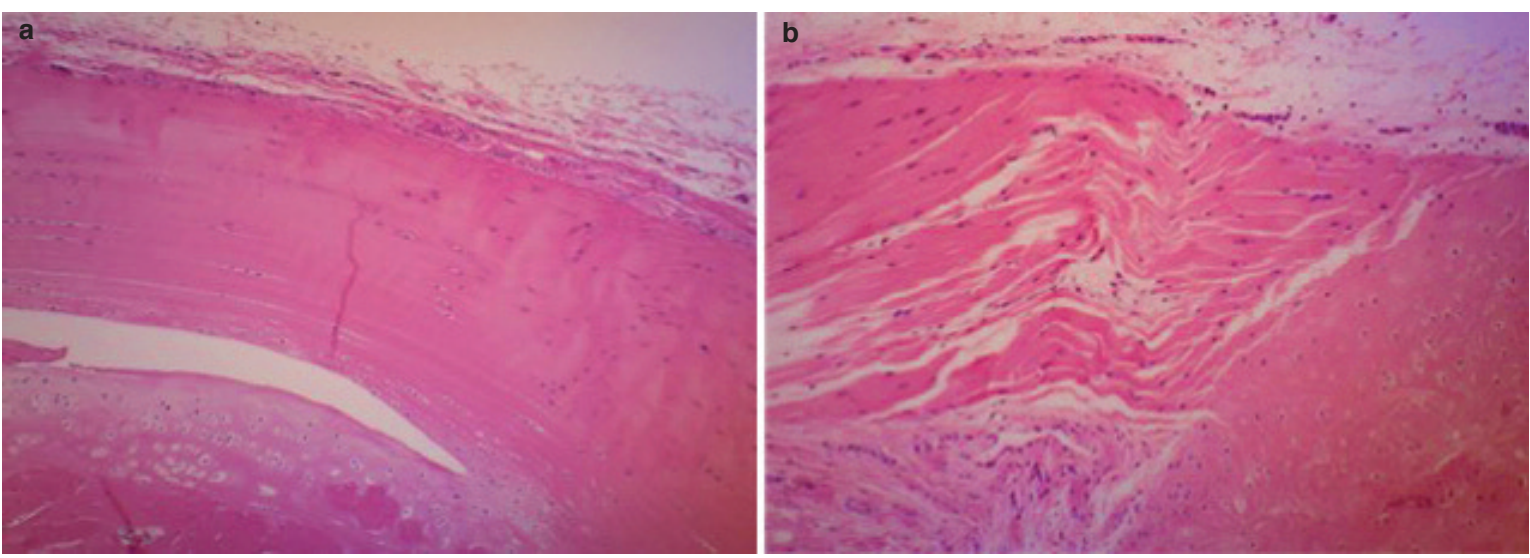

Figure 3. Histological sections of the tibial insertion sites of the patellar tendons stained with H\&E in a healthy rat (a) or in the tendinopathy rat (b) $(\times 100)$. Two major pathological findings were found with light microscopy at the tibial insertion site of the patellar tendons in the tendinopathy rats: (1) microtears in the body of the tendon and (2) several longitudinal disruptions between the layers of the fiber bundles of the tendon body, so-called laminations. 

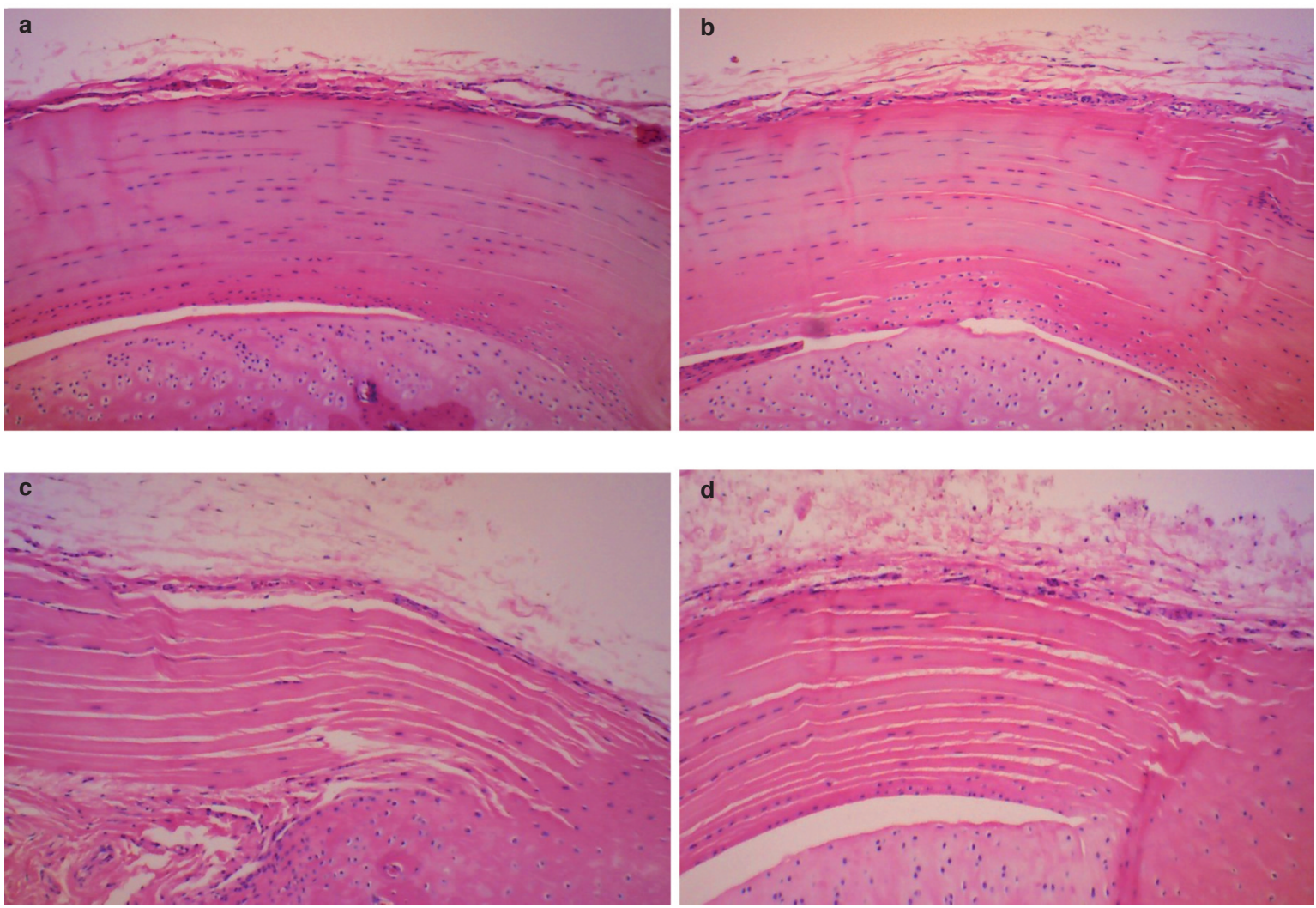

Figure 4. Histological sections of the tibial insertion sites of the patellar tendons stained with H\&E in the P10 group (a), in the P5 group (b), in the MN group (c) or in the control group (d) ( $\times 100)$. The number of microtears or laminations found in 5 rats of the P10 or the P5 groups was significantly lower than those in 5 rats of the MN or control groups $(P<0.05)$. No significant difference was observed in the number of microtears or laminations between the P10 group and the P5 group or among tendinopathy rats, $\mathrm{MN}$ group and control group.

Table II. Histopathological score for the histological sections located at the tibial insertion site of the patellar tendon.

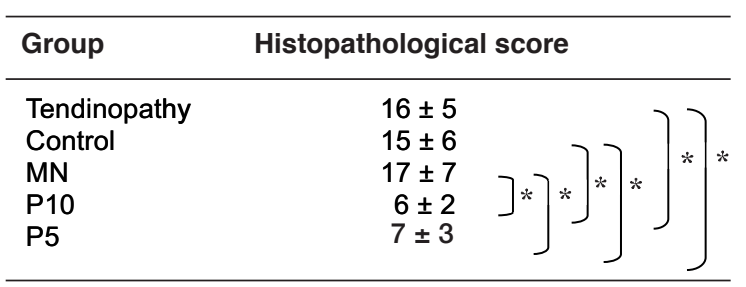

${ }^{*} p<0.05$

ties in the tendinopathy rats compared to those in the control or multiple needling groups; (2) the spontaneous locomotor activities in rats which were injected with autologous leukocyte-reduced PRP at the platelets concentration of $1.0 \times 10^{6} / \mu \mathrm{L}$ recovered up to the original healthy level at 2 days after injections and were then maintained, not decreased, for the following 15 days until the end of the study without any additional injections of PRP; (3) the spontaneous loco-
Table III. Cell number for apoptosis at the joint side of the tibial insertion site for the patellar tendon.

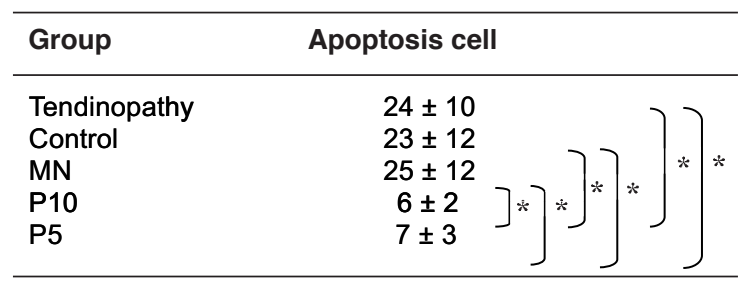

${ }^{*} p<0.05$

motor activities in the rats which were injected with autologous leukocyte-reduced PRP at the platelets concentration of $1.0 \times 10^{6} / \mu \mathrm{L}$ were significantly greater than those in the rats which were injected with PRP at the platelets concentration of $5.0 \times 10^{5} / \mu \mathrm{L}$ during the experimental periods for 16 days from day 2 until the end of the study; (4) the multiple dry-needling against the insertional tendinopathy site of the patellar tendon did not significantly increase the sponta- 

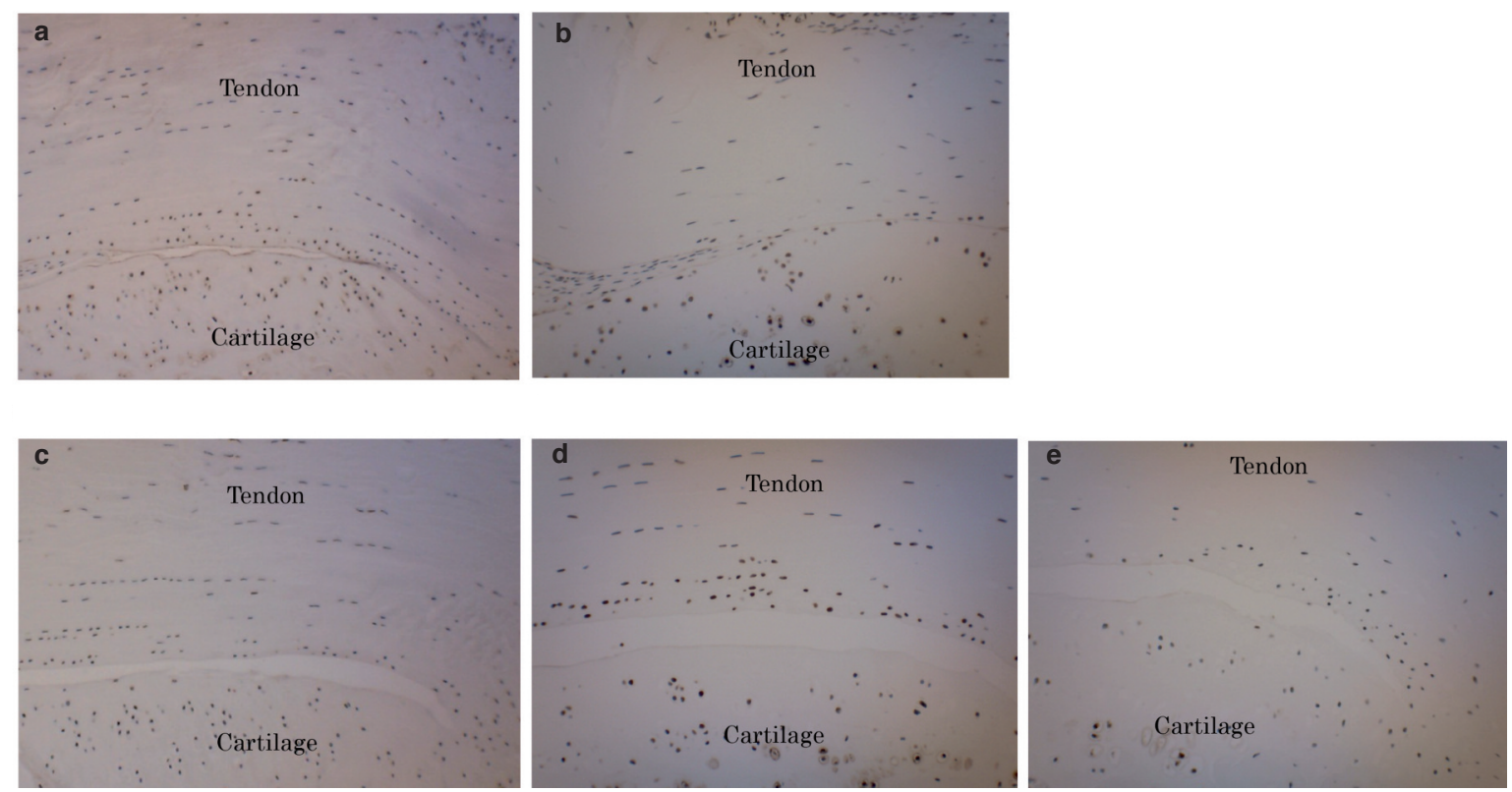

Figure 5. Histological sections of the tibial insertion sites of the patellar tendons prepared by TdT-mediated dUTP nick end labeling in the tendinopathy rats (a), in the P10 group (b), in the P5 group (c), in the MN group (d) or in the control group (e) (×220). The number of TUNEL-positive cells in the P10 or P5 groups was significantly lower than those in the MN or control groups at the joint side of the tibial insertional site of the patellar tendon $(P<0.05)$. No significant difference was observed in the number of TUNEL-positive cells between the P10 group and the P5 group or among tendinopathy rats, MN group and control group.

neous locomotor activities and no significant differences in the spontaneous locomotor activities were observed between the MN group and the control group; (5) the number of microtears or laminations observed in histological specimens at the tibial insertion site of the patellar tendon in tendinopathy rats decreased in the PRP group compared to those in the control or multiple needling groups at day 18; (6) the number of TUNEL-positive cells at the joint side of the tibial insertion site of the patellar tendon in the PRP group was lower than those in the control or multiple needing groups at day 18 ; $(7)$ no significant differences were observed in the histopathological scores or the number of TUNEL-positive cells between the P10 group and the P5 group or among tendinopathy rats, MN group and control group.

The findings of (1) and (2) indicated that an autologous leukocyte-reduced PRP injection was effective and rapid analgesic method in tendinopathy rats, since no remarkable tendinopathy-specific histological changes were observed in the rotator cuffs of the shoulders in tendinopathy rats. The present study for the first time demonstrated the analgesic effect of the autologous leukocyte-reduced PRP for the treatment of the tendinopathy in the rat model prepared by a repetitive running exercise on a rodent treadmill machine. Regarding the mechanism for the generation of the pain in the tendinopathy, there were several studies which showed that the abnormal capillary vessels with shunts were conformed around the ten- don, not intra tendon, in the tendinopathy ${ }^{34-36}$. It was suggested that the pain was generated from the free nerve endings, which were distributed along with the abnormal capillary vessels especially conformed in the tendinopathy, since the focal embolization of the abnormal capillary vessels immediately removed the pain in tendinopathy ${ }^{37}$. Therefore, it was speculated that the abnormal capillary vessels with free nerve endings were degradated by a reaction induced with an autologous leukocyte-reduced PRP, although no distinct capillary vessels were histologically found at the tibial insertional sites of the patellar tendon in our tendinopathy rats.

The findings of (2) and (3) indicated that the analgesic effect of PRP at the platelets concentration of $1.0 \times 10^{6} / \mu \mathrm{L}$ was greater than that of PRP at the platelets concentration of $5.0 \times 10^{5} / \mu \mathrm{L}$ in the tendinopathy rat model and that an injection of leukocytereduced $\mathrm{PRP}$ at the platelets concentration of $1.0 \times 10^{6} / \mu \mathrm{L}$ completely relieved the pain in the tendinopathy rat. It was estimated that the abnormal capillary vessels with free nerve endings were rapidly degradated enough to completely relief the pain by a reaction induced with a leukocyte-reduced PRP at the platelets concentration of $1.0 \times 10^{6} / \mu \mathrm{L}$. The finding of (7) indicated that the effects for the histological restoration and the apoptosis inhibition of leukocytereduced $\mathrm{PRP}$ at the platelets concentration of $1.0 \times 10^{6} / \mu \mathrm{L}$ or $5.0 \times 10^{5} / \mu \mathrm{L}$ were almost identical whereas the analgesic effects between them were 
different. Hence, it was proposed that the platelets concentration between $5.0 \times 10^{5} / \mu \mathrm{L}$ and $1.0 \times 10^{6} / \mu \mathrm{L}$ in leukocyte-reduced PRP was optimal for the treatment of the tendinopathy rat model.

The finding of (4) indicated that the multiple dryneedling against the tendinopathy site never relieved the pain and never improved the histopathological condition of the tendon. Dragoo also reported that 3 patients treated by dry-needling alone failed and required surgical intervention for the treatment of patellar tendinopathy ${ }^{7}$. Hence, it was demonstrated that there was no contribution in the multiple dry-needling for the treatment of the tendinopathy. However, it was necessary to needle and inject a PRP at several points in order to sufficiently infiltrate a PRP at the pathological tendinopathy site in clinic. Therefore, it was recommended that a small needle (30G) should be used and the number of the needling should be minimum for the injection of PRP in order to decrease pain and to prevent the tissue damage caused by multiple needling ${ }^{38}$.

The findings of (5) and (6) indicated that an autologous leukocyte-reduced PRP had a function to initiate the reparative reaction, to repair the microtears or the laminations observed in tendinopathy and to inhibit the apoptosis of the cells occurred in the tendinopathy. It was suggested that the platelets had numerous kinds of cytokines and chemokines as a package for the reparative reaction of the tissue and after the administration of leukocyte-reduced PRP the reparative reaction begun and progressed along with the cascades modulated with those cytokines or chemokines. The previous studies also showed the reparative function of PRP for the treatment of the tendinopathy ${ }^{16,17}$. It was suggested that a PRP therapy was indicated well for the tendinopathy since the conventional conservative therapies were not available for many cases of the tendinopathy and it was speculated that the reparative reaction in the tendinopathy was delayed and a tissue restoration was not progressed in the tendinopathy.

Limitations of the present study were small sample number, short experimental period, lack of biomechanical evaluation and that the present animal model reproduced the early-stage tendinopathy, not in the late-stage, which was characterized by fibrosis, lipid depositions, proteoglycan accumulation and calcification in the tendon. Zhang showed that an autologous platelet-rich clot releasate could not reverse the nontenogenic differentiation of tendon stem/progenitor cells which were pre-treatment in non-tenogenic media for 2 days in vitro model and the study suggested that the injection of PRP in clinics may not be able to effectively reverse the degenerative conditions of late-stage tendinopathy ${ }^{39}$. It was still uncertain whether the PRP therapy was effective for the latestage tendinopathy or not. Further examinations were necessary to dissolve those problems.

\section{Conclusion}

One injection of an autologous leukocyte-reduced PRP into the space between the patellar tendon and fat pad effectively relieved pain and initiated partial restoration of the patellar tendon in the tendinopathy rat model. The analgesic effects of the leukocyte-reduced PRP at the platelets concentration of $1.0 \times 10^{6} / \mu \mathrm{L}$ was greater than those at the half concentration of the platelets, although no difference was observed for the effect of the histological restoration between them. A multiple dry-needling showed no effects for the treatment of the tendinopathy. It was suggested that an administration of an autologous leukocyte-reduced PRP was clinically effective modality in treatment of tendinopathy.

\section{Abbreviations \\ PRP Platelet-rich plasma \\ NS Normal saline \\ EDTA Ethylenediamine tetra-acetic acid \\ H\&E Hematoxylin-eosin \\ TUNEL TdT-mediated dUTP nick end labeling}

\section{Key points}

Our goal was to assess the effects of pain-relieving and histological restoration for PRP therapy and to determine the optimal concentration of platelets in leukocytereduced PRP for the treatment of the tendinopathy.

We used patellar tendinopathy in a rat model prepared using a repetitive running exercise on a rodent treadmill machine with adequate controls.

We measured spontaneous locomotor activities of rats for $12 \mathrm{~h}$ every day for the assessment of a pain-relieving and we histologically analyzed a restoration of the tendon and an occurrence of apoptosis.

We demonstrated that a PRP therapy was effect for pain-relieving and histological restoration of the tendinopathy. The injections of a PRP at the platelets concentration of $1.0 \times 10^{6} / \mu \mathrm{L}$ completely relieved the pain and were more effective than those at the platelets concentration of $5.0 \times 10^{5} / \mu \mathrm{L}$ whereas there was no difference for the effects of histological restoration and apoptosis inhibition between them.

We estimated that the platelets concentration between $5.0 \times 10^{5} / \mu \mathrm{L}$ and $1.0 \times 10^{6} / \mu \mathrm{L}$ in leukocyte-reduced PRP was optimal for the treatment of the tendinopathy rat model.

The study results suggested that an administration of an autologous leukocyte-reduced PRP was clinically effective modality in the treatment of the tendinopathy.

\section{Conflicts of interests}

The Authors declare that they have no competing interests. 


\section{References}

1. Kaux JF, Forthomme B, Le Goff C, Crielaard JM,Croisier JL. Current Opinions on Tendinopathy. J Sports Sci Med. 2011;10 (2):238-253.

2. Mishra A, Pavelko T. Treatment of chronic elbow tendinosis with buffered platelet-rich plasma. Am J Sports Med. 2006;34 (11):1774-1778.

3. Peerbooms JC, Sluimer J, Bruijn DJ, Gosens T. Positive effect of an autologous platelet concentrate in lateral epicondylitis in a double-blind randomized controlled trial: Platelet-rich plasma versus corticosteroid injection with a 1-year follow-up. Am J Sports Med. 2010;38(2):255-262.

4. Gosens T, Peerbooms JC, van Laar W, den OudstenBL. Ongoing positive effect of platelet-rich plasma versus corticosteroid injection in lateral epicondylitis: A double-blind randomized controlled trial with 2-year follow-up. Am J Sports Med. 2011;39(6):1200-1208.

5. Thanasas C, Papadimitriou G, Charalambidis C, Paraskevopoulos I, Papanikolaou A.Platelet-rich plasma versus autologous whole blood for the treatment of chronic lateral elbow epicondylitis: a randomized controlled clinical trial. Am J Sports Med. 2011;39(10):2130-2134

6. Vetrano M, Castorina A, Vulpiani MC, Baldini R, Pavan A, Ferretti $A$. Platelet-rich plasma versus focused shock waves in the treatment of jumper's knee in athletes. Am J Sports Med. 2013;41(4):795-803.

7. Dragoo JL, Wasterlain AS, Braun HJ, Nead KT. Platelet-rich plasma as a treatment for patellar tendinopathy: a doubleblind, randomized controlled trial. Am J Sports Med. 2014; 42(3):610-618

8. Murawski CD, Smyth NA, Newman H, Kennedy JG.A single platelet-rich plasma injection for chronic midsubstanceachilles tendinopathy: a retrospective preliminary analysis. Foot Ankle Spec. 2014;7(5):372-376.

9. Filardo G, Kon E, Di Matteo B, et al. Platelet-rich plasma injections for the treatment of refractory Achilles tendinopathy: results at 4 years. Blood Transfus. 2014;12(4):533-540.

10. Dallaudière B, Pesquer L, Meyer $P$, et al. Intratendinous injection of platelet-rich plasma under US guidance to treat tendinopathy: a long-term pilot study. J Vasc Interv Radiol. 2014;25 (5):717-723.

11. Kaux JF, Bruyere O, Croisier JL, Forthomme B, Le Goff C Crielaard JM. One-year follow-up of platelet-rich plasma infiltration to treat chronic proximal patellar tendinopathies.Acta Orthop Belg. 2015;81(2):251-256.

12. Guelfi M, Pantalone A, Vanni D, Abate M, Guelfi MG, Salini V. Long-term beneficial effects of platelet-rich plasma for non-insertional Achilles tendinopathy. Foot Ankle Surg. 2015;21 (3):178-181.

13. deVos RJ, Weir A, van Schie HT, et al. Platelet-rich plasma injection for chronic Achilles tendinopathy: A randomized controlled trial. JAMA. 2010;303(2):144-149.

14. de Jones S, de Vos RJ, Weir A, et al. One-year follow-up of platelet-rich plasma treatment in chronic Achilles tendinopathy: A double-blind randomized placebo-controlled trial. Am J Sports Med. 2011;39(8):1623-1629.

15. Kaux JF, Drion P, Croisier JL,Crielaard JM. Tendinopathies and platelet-rich plasma (PRP): from pre-clinical experiments to therapeutic use. J Stem Cells Regen Med. 2015;11(1):717.

16. Dallaudière B, Lempicki M, Pesquer L, et al. Efficacy of intratendinous injection of platelet-rich plasma in treating tendinosis: comprehensive assessment of a rat model. Eur Radiol. 2013;23(10):2830-2837.
17. Chen L, Liu JP, Tang KL, et al. Tendon derived stem cells promote platelet-rich plasma healing in collagenase-induced rat Achilles tendinopathy. Cell Physiol Biochem. 2014;34 (6): 2153-2168.

18. Flatow EL, Nasser P, Lee L, Schaffler MB, Jepsen KJ. Overestimation of the degradation state in fatigue loaded tendon due to transient effects. Trans Orthop Res Soc. 2002;27:621.

19. Wang VM, Laudier D, Tsai CW, Jepsen KJ, Schaffler MB, Flatow EL. Imaging normal and damaged tendons: development and application of novel tissue processing techniques. Trans Orthop Res Soc. 2005;30:321.

20. Lee H, Wang VM, Laudier DM, Schaffler MB, Flatow EL. A novel in vivo model of tendon fatigue damage accumulation. Trans Orthop Res Soc. 2006;31:1058.

21. Lake SP, Ansorge HL, Soslowsky LJ. Animal models of tendinopathy. Disabil Rehabil. 2008;30(20-22):1530-1541.

22. Dallaudiere B, Louedec L, Lenet MP, et al.The molecular systemic and local effects of intra-tendinous injection ofPlatelet Rich Plasma in tendinosis: preliminary results on a rat model withELISA method. Muscles Ligaments Tendons J. 2015;5(2): 99-105.

23. Yoshida M, Funasaki H, Kubota M, Marumo K. Therapeutic effects of high molecular weight hyaluronan injections for tendinopathy in a rat model. J Orthop Sci. 2015;20:186-195.

24. McCarrel TM, Minas T, Fortier LA. Optimization of leukocyte concentration in platelet-rich plasma for the treatment of tendinopathy. e143J Bone Joint Surg Am. 2012;94:1-8.

25. Boswell SG, Schnabel LV, Mohammed HO, Sundman EA, Minas T, Fortier LA. Increasing platelet concentrations in leukocyte-reduced platelet-rich plasma decrease collagen gene synthesis in tendons. Am J Sports Med. 2014;42(1):42-9.

26. Giusti I, D'Ascenzo S, Manco A, et al. Platelet concentration in platelet-rich plasma affects tenocyte behavior in vitro. BioMed Res Int. 2014;2014:630870.

27. Dragoo JL, Braun HJ, Durham JL, et al. Comparison of the acute inflammatory response of two commercial platelet-rich plasma systems in healthy rabbit tendons. Am J Sports Med. 2012;40:1274-1281.

28. Padulo J, Oliva F, Frizziero A, Maffulli N. Muscles, Ligaments and Tendons Journal. Basic principles and recommendations in clinical and field science research: 2016 update. MLTJ. 2016;6 (1):1-5.

29. Yinghua X, George ACM. The basic science of tendinopathy. ClinOrthopRelat Res. 2008;466:1528-1538.

30. Masuo Y, Matsumoto Y, Morita S, Noguchi J. A novel method for counting spontaneous motor activity in the rat. Brain Research Protocols. 1997;1:321-326.

31. Hara J, Beuckmann TC, Nambu T, et al. Genetic ablation of orexin neurons in mice results in narcolepsy, hypophagia, and obesity. Neuron. 2001;30:345-354.

32. Shibakusa T, Iwaki Y, Mizunoya W, et al. The physiological and behavioral effects of subchronicintracisternal administration of TGF- $\beta$ in rats: comparison with the effects of CRF. Biomedical Research. 2006;27:297-305.

33. Watabiki T, Kiso T, Tsukamoto M, Aoki T, Matsuoka N. Intrathecal administration of AS1928370, a transient receptor potential vanilloid 1 antagonist, attenuates mechanical allodynia in a mouse model of neuropathic pain. Biol Pharm Bull. 2011;34:1105-1108.

34. Alfredson $\mathrm{H}$, Ohberg $\mathrm{L}$, Forsgren $\mathrm{S}$. Is vasculo-neural ingrowth the cause of pain in chronic Achilles tendinosis? An investigation using ultrasonography and colour Doppler, immunohistochemistry, and diagnostic injections.Knee Surg Sports Traumatol Arthrosc.2003;11(5):334-338.

35. Ohberg L, Alfredson $\mathrm{H}$. Effects on neovascularisation behind 
the good results with eccentric training in chronic mid-portion Achilles tendinosis? Knee Surg SportsTraumatol Arthrosc. 2004;12(5):465-470.

36. OkunoY, Nakamura-Ishizu A, Otsu K, Suda T, Kubota Y Pathological neoangiogenesis depends on oxidative stress regulation by ATM.Nat Med. 2012;18(8):1208-1216.

37. Okuno Y, Matsumura N, Oguro S. Transcatheter arterial embolization using imipenem/cilastatin sodium for tendinopathy and enthesopathy refractory to nonsurgical management. J Vasc Interv Radiol. 2013;24(6):787-792.

38. Bausset O, Magalon J, Giraudo L, et al. Impact of local anaesthetics and needle calibres used for painless PRP injections on platelet functionality. Muscles Ligaments Tendons J. 2014;4(1):18-23.

39. Zhang J, Wang JH. PRP treatment effects on degenerative tendinopathy - an in vitro model study. Muscles Ligaments Tendons J. 2014;8;4(1):10-7. eCollection 2014. 